\title{
Significant improvement of biocompatibility of polypropylene mesh for incisional hernia repair by using poly- $\varepsilon$-caprolactone nanofibers functionalized with thrombocyte-rich solution
}

\author{
This article was published in the following Dove Press journal: \\ International Journal of Nanomedicine \\ I April 2015 \\ Number of times this article has been viewed
}

\author{
Martin Plencner ${ }^{1,2}$ \\ Eva Prosecká ${ }^{1,2}$ \\ Michala Rampichová ${ }^{2,3}$ \\ Barbora East ${ }^{4}$ \\ Matej Buzgo 2,3 \\ Lucie Vysloužilová ${ }^{3}$ \\ Jiří Hoch ${ }^{4}$ \\ Evžen Amler ${ }^{1,2,5}$ \\ 'Institute of Biophysics, 2nd Faculty \\ of Medicine, Charles University \\ in Prague, Prague, ${ }^{2}$ Laboratory of \\ Tissue Engineering, Institute of \\ Experimental Medicine, Academy \\ of Sciences of the Czech Republic, \\ Prague, ${ }^{3}$ University Center for Energy \\ Efficient Buildings (UCEEB), The \\ Czech Technical University in Prague, \\ Bustehrad, ${ }^{4}$ Department of Surgery, \\ 2nd Faculty of Medicine, Charles \\ University in Prague, Prague, ${ }^{5}$ Faculty \\ of Biomedical Engineering, Czech \\ Technical University in Prague, Kladno, \\ Czech Republic
}

\begin{abstract}
Incisional hernia is the most common postoperative complication, affecting up to $20 \%$ of patients after abdominal surgery. Insertion of a synthetic surgical mesh has become the standard of care in ventral hernia repair. However, the implementation of a mesh does not reduce the risk of recurrence and the onset of hernia recurrence is only delayed by 2-3 years. Nowadays, more than 100 surgical meshes are available on the market, with polypropylene the most widely used for ventral hernia repair. Nonetheless, the ideal mesh does not exist yet; it still needs to be developed. Polycaprolactone nanofibers appear to be a suitable material for different kinds of cells, including fibroblasts, chondrocytes, and mesenchymal stem cells. The aim of the study reported here was to develop a functionalized scaffold for ventral hernia regeneration. We prepared a novel composite scaffold based on a polypropylene surgical mesh functionalized with poly- $\varepsilon$-caprolactone (PCL) nanofibers and adhered thrombocytes as a natural source of growth factors. In extensive in vitro tests, we proved the biocompatibility of PCL nanofibers with adhered thrombocytes deposited on a polypropylene mesh. Compared with polypropylene mesh alone, this composite scaffold provided better adhesion, growth, metabolic activity, proliferation, and viability of mouse fibroblasts in all tests and was even better than a polypropylene mesh functionalized with PCL nanofibers. The gradual release of growth factors from biocompatible nanofiber-modified scaffolds seems to be a promising approach in tissue engineering and regenerative medicine.
\end{abstract}

Keywords: nanofibers, growth factors, polypropylene mesh, hernia regeneration, in vitro

\section{Introduction}

Incisional hernia is the most common postoperative complication, affecting up to $20 \%$ of patients after a midline incision. ${ }^{1,2}$ The 10-year cumulative rate of recurrence after incisional hernia repair is at an unacceptable level of $63 \%$ for suture repair and $32 \%$ for prosthetic repair. ${ }^{3}$ The cumulative incidence of re-operation after incisional hernia repair, with or without a surgical mesh, has exhibited a linear rise over the years. It has been found that implantation of a mesh does not reduce the risk of recurrence and only delays the onset of hernia recurrence by $2-3$ years. ${ }^{4}$

Repairs to a large incisional defect often require the use of a prosthetic implant, which can cause various long-term complications such as adhesion formation, graft infection/rejection, fistula formation, and hernia recurrence. ${ }^{5-8}$ Synthetic meshes have become the standard of care in ventral hernia repair. ${ }^{9}$ Incorporating polymeric meshes during hernia repair has been demonstrated to reduce recurrence rates, minimize pain, and improve patient outcomes postoperatively in general. ${ }^{3,10}$ More than 100 surgical
Correspondence: Martin Plencner Laboratory of Tissue Engineering, Institute of Experimental Medicine, Academy of Sciences of the Czech Republic, v.v.i., Vídeňská 1083, 14240 Prague, Czech Republic

Tel/Fax +420 296442387

Email martin.plencner@gmail.com 
meshes have now been designed for use in hernia repair procedures. ${ }^{11}$ However, no single mesh has yet demonstrated the ability simultaneously to promote host-tissue remodeling and high-strength repair of abdominal wall defects. ${ }^{12}$ The ideal mesh does not yet exist, and still needs to be developed.

In general, non-resorbable synthetic meshes are made of polypropylene (PP; Prolene ${ }^{\circledR}$, Marlex $^{\circledR}$, Vypro $^{\circledR}$, ProLite $\left.^{\mathrm{TM}}\right)$, polyesters $\left(\right.$ Dacron $^{\circledR}$, Mersilene $\left.^{\mathrm{TM}}\right)$, and expanded polytetrafluoroethylene (ePTFE; Gore-Tex ${ }^{\circledR}$ ). The group of absorbable synthetics includes glycolic acid (Vicryl ${ }^{\circledR}$ ), polyglycolic acid $\left(\right.$ Dexon $\left.^{\circledR}\right)$, and carboxycellulose $\left(\right.$ Seprafilm $\left.^{\circledR}\right)$. Absorbable prostheses are generally applied for temporary abdominal closure, or are used in conjunction with permanent synthetics. ${ }^{11}$ Many different ways of classifying surgical meshes have been presented. ${ }^{13,14}$

In addition to synthetic meshes, there are a few commercially-available biological meshes with appropriate biocompatible features - for example, derived bovine pericardium (Tutomesh $\left.{ }^{\circledR}\right),{ }^{15}$ acellular porcine dermis (Permacol $\left.{ }^{\mathrm{TM}}\right),{ }^{16}$ and acellular analog of human dermis (AlloDerm $\left.{ }^{\circledR}\right) .{ }^{17}$ However, the use of xenografts is nowadays on the decline, and the production of this type of mesh is limited by the extremely high cost.

An optimal mesh for use in ventral hernia regeneration should meet a number of criteria. The mesh has to be chemically inert, biocompatible, with sufficient biomechanical properties, and it should not cause inflammation or an allergic response. One way to improve the properties of the meshes used until now is to combine them with other tissue-engineered materials, or to replace the meshes completely with these materials. Highly porous scaffolds with interconnected pores are favored in tissue-engineering applications. ${ }^{18}$ Much attention has been paid to the application of biodegradable microfibers and nanofibers. Nanofiber scaffolds produced by electrospinning contain a large number of interconnected pores and fibers in the diameter of the extracellular matrix (ECM). ${ }^{19}$ The ECM plays a pivotal role in controlling cell behaviors, such as adhesion, proliferation, migration, and differentiation. ${ }^{20}$

Nanofibers can be produced from materials that are biocompatible and biodegradable, such as poly- $\varepsilon$-caprolactone (PCL), ${ }^{21}$ poly(lactic-co-glycolic) acid (PLGA), ${ }^{21}$ and chitosan. ${ }^{22}$ PCL has frequently been chosen for electrospinning, because it is a US Food and Drug Administration (FDA)-approved material and has been shown to support the attachment and growth of chondrocytes, ${ }^{23}$ osteoblasts, ${ }^{24,25}$ smooth muscle cells, ${ }^{26}$ fibroblasts, ${ }^{24,27-29}$ myoblasts, ${ }^{30}$ and mesenchymal stem cells (MSCs). ${ }^{31}$ PCL appears to be a suitable material for scaffold preparation for reparative surgery. It has been used in wound healing, ${ }^{32}$ and could be used in combination with a surgical mesh in ventral hernia regeneration.

Nanofibers have a high surface-area-to-volume ratio, which allows adsorption and high immobilization of cells as well as blood derivatives such as platelet-rich plasma (PRP). ${ }^{33}$ The proliferative effect of thrombocytes and their fraction present in PRP on chondrocytes and on MSCs has been confirmed in our laboratory. ${ }^{23,34}$ This observation was in accordance with previous reports. ${ }^{35}$ PRP contains high levels of growth factors (GFs), such as platelet-derived growth factor, transforming growth factor-beta, insulin-like growth factor-1, epidermal growth factor, and vascular endothelial growth factor, as well as the plasma components fibrin, fibronectin, and vitronectin. ${ }^{36,37}$ GFs present in platelets have been shown to be effective in promoting wound healing and regeneration. ${ }^{38}$ In the study reported here, we used thrombocytes concentrated in a buffer solution (thrombocyte-rich solution [TRS]). An advantage of TRS is that it does not contain leucocytes or erythrocytes, which are present in plasma. This minimizes the immune response when it is applied.

The aim of this study was to develop a functionalized scaffold for ventral hernia regeneration. We prepared a novel composite scaffold based on a PP surgical mesh functionalized with PCL nanofibers and adhered thrombocytes. The effect of the released GFs and also the effect of the PCL nanofibers themselves on fibroblast growth and proliferation were evaluated in vitro.

\section{Materials and methods Scaffold preparation}

PCL nanofibers were prepared by an electrospinning method from PCL with molecular weight (MW) 45,000 (Sigma-Aldrich, St Louis, MO, USA). ${ }^{39}$ Electrospinning was performed from a $14 \%$ solution of PCL dissolved in chloroform:ethanol at a ratio of 8:2. A high-voltage source generated voltages of up to $50 \mathrm{kV}$, and the polymer solution was connected to a high-voltage source. Electrospun nanofibers were deposited on the grounded collecting electrode. A PP surgical mesh (Prolene, Ethicon Inc, Somerville, NJ, USA) was coated with PCL nanofibers. Prolene was attached to the grounded collector, and PCL nanofibers were deposited on the mesh from each side (Figure 1).

\section{Thrombocyte-rich solution preparation}

First, PRP was obtained from the Hematology Service of the General Teaching Hospital, Prague, Czech Republic. 


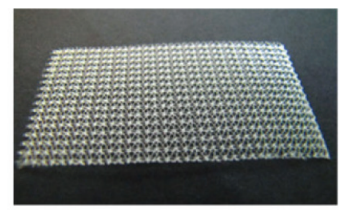

PP
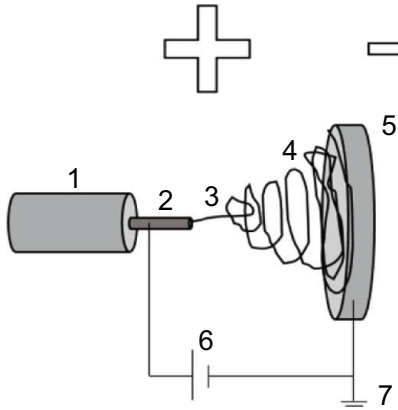

Electrospinning

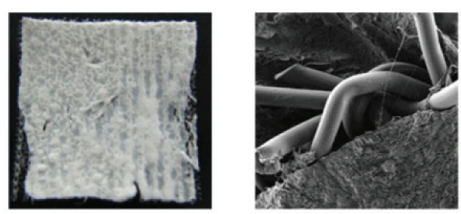

$P P+P C L$
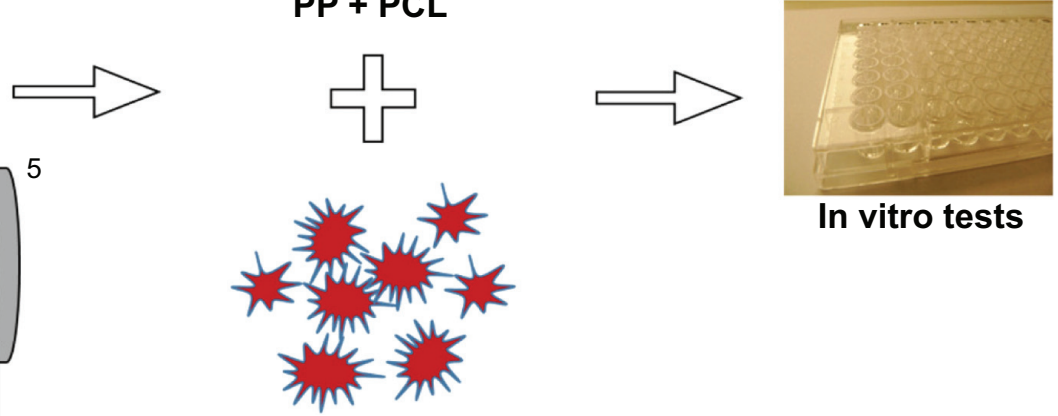

In vitro tests

Figure I Methodology of the scaffold fabrication. Poly-ع-caprolactone (PCL) nanofibers were prepared by an electrospinning method. Electrospun nanofibers were deposited on a polypropylene (PP) surgical mesh, which was attached to the grounded collecting electrode from each side. PP covered with PCL nanofibers was cut into round patches of $6 \mathrm{~mm}$ in diameter, sterilized, and immersed in thrombocytes-rich solution (TRS) for 2 hours. The nonadhered thrombocytes were removed by rinsing twice in phosphatebuffered saline. The composite scaffolds were placed in a new well, seeded with 3T3 fibroblasts and tested in vitro.

Notes: (I) Syringe and metering pump, (2) needle serving as the electrode, (3) stable part of the jet, (4) whipping/coiling zone, (5) collector covered with PP, (6) ground, and (7) high-voltage supply.

PRP (volume $200 \mathrm{~mL}$, thrombocyte concentration $106 \times 10^{7}$ thrombocytes $/ \mathrm{mL}$ ) was centrifuged $(2,250 \mathrm{~g}, 15$ minutes), then the supernatant was discarded, and the resulting thrombocytes were washed in a washing buffer $(113 \mathrm{mM} \mathrm{NaCl}$, $4.3 \mathrm{mM} \mathrm{K}_{2} \mathrm{HPO}_{4}, 4.3 \mathrm{mM} \mathrm{Na}_{2} \mathrm{HPO}_{4}, 24.4 \mathrm{mM} \mathrm{NaH}_{2} \mathrm{PO}_{4}$, and $5.5 \mathrm{mM}$ glucose, $\mathrm{pH}$ 6.5) as described by Baenziger et al. ${ }^{40}$ Contaminating leukocytes and erythrocytes were removed by further centrifugation ( $120 \mathrm{~g}, 7$ minutes). The thrombocytes were washed until the leukocytes and erythrocytes contained in them were removed. The thrombocytes were pelleted by centrifugation $(2,000 \mathrm{~g}, 15$ minutes $)$, and were then washed once before finally being resuspended in $10 \mathrm{~mL}$ of a $\mathrm{pH} 7.5$ buffer (109 mM NaCl, $4.3 \mathrm{mM} \mathrm{K}_{2} \mathrm{HPO}_{4}, 16 \mathrm{mM} \mathrm{Na}_{2} \mathrm{HPO}_{4}$, $8.3 \mathrm{mM} \mathrm{NaH}_{2} \mathrm{PO}_{4}$, and $5.5 \mathrm{mM}$ glucose). The TRS was stored and shaken in centrifuge tubes at $22^{\circ} \mathrm{C}$.

\section{Composite scaffold preparation}

Before cell seeding, PCL nanofibers and Prolene coated with PCL nanofibers were cut into round patches $6 \mathrm{~mm}$ in diameter and sterilized using ethylene oxide at $37^{\circ} \mathrm{C}$. The scaffolds were used 1 week after sterilization, in order to air out possible remnants of ethylene oxide. The scaffolds were immersed in TRS $\left(106 \times 10^{6}\right.$ thrombocytes $\left./ \mathrm{mL}\right)$ for 2 hours to enable adhesion of thrombocytes (Figure 1). ${ }^{23}$ After the incubation time, nonadhered platelets were removed by rinsing twice in phosphate-buffered saline (PBS; pH 7.4). Scaffolds without adhered thrombocytes were incubated in
PBS (pH 7.4) for 2 hours. Then the composite scaffold was placed in a new well and seeded with $3 \mathrm{~T} 3$ fibroblasts. GFs were released from the thrombocytes adhered to the nanofibers over a period of 1-14 days. ${ }^{34}$

\section{Scanning electron microscopy and stereological analysis of the scaffolds}

Nanofibers were sputter-coated with a layer of gold approximately $60 \mathrm{~nm}$ in thickness using a Polaron SC510 Sputter Coater (Quorum Technologies Ltd, East Grinstead, UK). The samples were examined in an Aquasem scanning electron microscope (Tescan, Brno, Czech Republic) in secondary electron mode at $15 \mathrm{kV}$.

The electrospun scaffolds were characterized in terms of fiber diameter and pore size using mathematical stereological methods, as described in detail previously. ${ }^{41}$ Briefly, the stereological parameters were measured from arbitrarily selected sections of the scanning electron microscopy (SEM) images, using Ellipse software (v 2001; ViDiTo, Kosice, Slovak Republic). The distribution of the fiber diameters and pore sizes was determined quantitatively from 200 measurements.

\section{Cell cultivation and seeding}

The mouse 3T3 fibroblast cells (line 3T3-Swiss albino CCL-92 ${ }^{\mathrm{TM}}$, American Type Culture Collection [ATCC], Manassas, VA, USA) were routinely maintained in a 
humidified incubator with an atmosphere of $5 \% \mathrm{CO}_{2}$ in air at $37^{\circ} \mathrm{C}$ with fresh medium added every 2 days. Cells were cultured in Dulbecco's Modified Eagle's Medium (DMEM; PAN-Biotech GmbH, Aidenbach, Germany) supplemented with 10\% fetal bovine serum (PAA Laboratories $\mathrm{GmbH}$, Pasching, Austria) and penicillin/streptomycin (100 IU/mL and $100 \mu \mathrm{g} / \mathrm{mL}$, respectively; Sigma-Aldrich). When the cells reached $80 \%-90 \%$ confluence, they were suspended using trypsin-ethylenediaminetetraacetic acid (EDTA; PAA Laboratories $\mathrm{GmbH}$ ). The number of cells was determined using light microscopy. To detect the metabolic activity, proliferation, and viability, fibroblasts were seeded on the scaffolds at a density of $3 \times 10^{3}$ cells $/ \mathrm{cm}^{2}$ and, to determine cell adhesion, at a density of $3 \times 10^{4}$ cells $/ \mathrm{cm}^{2}$. To eliminate any contribution from non-adherent cells, each scaffold was transferred to a new well on the plate before any in vitro tests. From each well, which contained $300 \mu \mathrm{L}$ of medium, a volume of $150 \mu \mathrm{L}$ of the medium was exchanged every 2nd day.

\section{Detection of cell adhesion by 3,3'- Dihexyloxacarbocyanine iodide staining} Staining with a 3,3'-Dihexyloxacarbocyanine iodide (DiOC6; Invitrogen, Carlsbad, CA, USA) fluorescent probe was used to detect adhesion of cells on the scaffolds. Samples were fixed with frozen methyl alcohol $\left(-20^{\circ} \mathrm{C}\right)$ for 10 minutes, then were rinsed with PBS followed by DiOC6 $(0.1-1.0 \mu \mathrm{g} / \mathrm{mL}$ in PBS, pH 7.4). After 45 minutes' incubation at room temperature (RT), the samples were rinsed with PBS (pH 7.4), then propidium iodide $(5 \mu \mathrm{g} / \mathrm{mL}$ in $\mathrm{PBS}, \mathrm{pH} 7.4)$ was added for 10 minutes. The samples were rinsed with PBS ( $\mathrm{pH} 7.4$ ) again before being visualized using an LSM 5 DUO confocal microscope (Carl Zeiss AG, Oberkochen, Germany; $\lambda_{\text {exc }}=484 \mathrm{~nm}$ and $\lambda_{\text {em }}=482-497 \mathrm{~nm}$ ). The areas of adhered cells were counted with Ellipse software. For each scaffold, an area of 100 cells was measured and averaged.

\section{Cell metabolic activity analysis} by 3-[4,5-dimethylthiazol-2-yl]-2,5diphenyltetrazolium bromide test

Cell metabolic activity was measured using the 3-[4,5dimethylthiazol-2-yl]-2,5-diphenyltetrazolium bromide (MTT) test. MTT ( $50 \mu \mathrm{L}, 1 \mathrm{mg} / \mathrm{mL}$; Sigma-Aldrich) in PBS ( $\mathrm{pH} 7.4$ ) was added to $150 \mu \mathrm{L}$ of the sample medium and incubated for 4 hours at $37^{\circ} \mathrm{C}$. Using mitochondrial dehydrogenase of normally metabolizing cells, the MTT was reduced to purple formazan. The formazan crystals were solubilized with $100 \mu \mathrm{L}$ of $50 \% N, N$-dimethylformamide in $20 \%$ sodium dodecyl sulfate at $\mathrm{pH}$ 4.7. The results were examined by spectrophotometry in an enzyme-linked immunosorbent assay (ELISA) microplate reader (ELx800 ${ }^{\mathrm{TM}}$; BioTek, Winooski VT, USA) at $570 \mathrm{~nm}$ (reference wavelength $690 \mathrm{~nm}$ ). The metabolic activity of $3 \mathrm{~T} 3$ fibroblasts on a scaffold was tested on Days 1, 3, 7, 10, and 14 .

\section{Cell proliferation analysis by PicoGreen ${ }^{\circledR}$ assay}

The second proliferation analysis was carried out using an Invitrogen PicoGreen assay kit. The proliferation of 3T3 fibroblasts on the scaffolds was tested on Days 1, 3, 7, 10, and 14. To process the material for analysis of the DNA content, each scaffold was replaced in a vial with $500 \mu \mathrm{L}$ of cell lysis solution $\left(0.2 \% \mathrm{v} / \mathrm{v}\right.$ Triton $^{\mathrm{TM}} \mathrm{X}-100$, $10 \mathrm{mM}$ tris(hydroxymethyl)aminomethane [Tris; $\mathrm{pH}$ 7.0], $1 \mathrm{mM}$ EDTA). To prepare the cell lysate, the samples were processed through a total of three freeze/thaw cycles; the scaffold sample was first frozen at $-70^{\circ} \mathrm{C}$ and thawed at RT. Between each freeze-thaw cycle, the scaffolds were roughly vortexed. The prepared samples were stored at $-70^{\circ} \mathrm{C}$ until analysis. To quantify the number of cells on the scaffolds, a cell-based standard curve was prepared using samples with a known number of cells (range $100-5 \times 10^{5}$ cells). The DNA content was determined by mixing $100 \mu \mathrm{L}$ PicoGreen reagent and $100 \mu \mathrm{L}$ of the DNA sample. The samples were loaded in triplicate and the florescence intensity was measured on a multimode microplate reader (Synergy HT, BioTek; $\lambda_{\mathrm{ex}}=480-500 \mathrm{~nm}, \lambda_{\mathrm{em}}=520-540 \mathrm{~nm}$ ).

\section{Viability of cells seeded on scaffolds by live/dead staining}

To detect cell viability, live/dead cell staining was performed. The cell viability of 3T3 fibroblasts on the scaffold was tested on Days 1, 3, 7, 10, and 14. The cells were stained with $2^{\prime}, 7^{\prime}$-Bis(2carboxyethyl)-5(6)-carboxyfluorescein acetoxymethyl ester (BCECF-AM; storage solution $1 \mathrm{mg} / \mathrm{mL}$ in dimethyl sulfoxide), and were finally diluted $1: 100$ in a serum-free medium (Sigma-Aldrich) and propidium iodide (5 $\mu \mathrm{g} / \mathrm{mL}$ in PBS, pH 7.4; Sigma-Aldrich). BCECF-AM was added to the scaffolds and incubated for 45 minutes at $37^{\circ} \mathrm{C}$ and $5 \% \mathrm{CO}_{2}$, then subsequently rinsed in PBS (pH 7.4). After rinsing with PBS ( $\mathrm{pH} 7.4$ ), propidium iodide was added for 10 minutes, then the scaffolds were rinsed again with PBS ( $\mathrm{pH}$ 7.4) and visualized using the Zeiss LSM 5 DUO confocal microscope (BCECF-AM $\lambda_{\text {ex }}=488 \mathrm{~nm}$ and $\lambda_{\mathrm{em}}=505-535 \mathrm{~nm}$; propidium iodide $\lambda_{\mathrm{ex}}=543 \mathrm{~nm}$ and $\left.\lambda_{\mathrm{em}}=630-700 \mathrm{~nm}\right)$.

"BCECF-AM" is an intracellular fluorescent $\mathrm{pH}$ indicator, which is hydrolysed to $2^{\prime}, 7^{\prime}$-Bis(2 carboxyethyl)-5(6)- 
carboxyfluorescein (BCECF) by cytosolic esterases. Thus, only live cells contribute to the staining results (green color). Propidium iodide binds to double-stranded DNA, but it can only cross the plasma membranes of nonviable cells (red color). For each scaffold, the number of live/dead cells was counted with Ellipse software and the number of cells cultivated on the same group of scaffold was averaged. Viability was calculated as the percentage of live cells from the total cell count per unit area.

\section{Cell proliferation analysis by colorimetric immunoassay}

The proliferation activity of 3T3 fibroblasts seeded on the scaffolds was determined using a colorimetric immunoassay based on measurements of 5-bromo-2'-deoxyuridine (BrdU), which is incorporated during DNA synthesis (Cell proliferation ELISA, BrdU [colorimetric], Hoffman-La Roche Ltd, Basel, Switzerland). The assay was performed according to the manufacturer's instructions. Briefly, on Days 1, 3, 7, 10, and 14, $100 \mu \mathrm{L}$ of BrdU-labeling solution was added to each well containing a scaffold and was allowed to incorporate into the cells in a $\mathrm{CO}_{2}$ incubator (Shellab SS-2306; Sheldon Manufacturing, Inc., Cornelius, OR, USA) at $37^{\circ} \mathrm{C}$ for 2 hours. Subsequently, the supernatant in each well was removed, and the scaffolds were incubated with FixDenat solution to fix the cells and denature the DNA at RT for 30 minutes. The supernatant was removed and, subsequently, $100 \mu \mathrm{L}$ anti-BrdU peroxidase (dilution ratio $=1: 100$ ) was added and kept at RT for 60 minutes. After removing the unbound antibody conjugate, $100 \mu \mathrm{L}$ of substrate solution was added and the solution was allowed to stand for 4 minutes. Following this, the reaction was completed by adding $25 \mu \mathrm{L}$ of $\mathrm{H}_{2} \mathrm{SO}_{4}$ solution $(1 \mathrm{M})$. Then, $100 \mu \mathrm{L}$ of the solution was transferred to a 96 -well plate and measured within 5 minutes at $450 \mathrm{~nm}$ with a reference wavelength of $690 \mathrm{~nm}$, using an ELx800 ${ }^{\mathrm{TM}}$ ELISA microplate reader (BioTek). The blank corresponded to a scaffold without cells, with or without BrdU.

\section{Statistics}

Quantitative data are presented as mean \pm standard deviation. For the in vitro tests, average values were determined from four independently prepared samples. The results were evaluated statistically, using one-way analysis of variance and the Student-Newman-Keuls method. The levels of significance were set at 0.001 and 0.05 .

\section{Results \\ Scanning electron microscopy and stereological analysis of the scaffolds}

The functionalized mesh was prepared by attaching the PP mesh onto the collector before the electrospinning process. PCL nanofibers were deposited on the surface of the PP mesh. The PP mesh with nanofibers was exposed in an aqueous environment for 2 weeks without any visual effect on the functionalized mesh. Three types of functionalized scaffolds were examined using SEM in secondary electron mode. Samples of the PP mesh (Figure 2B) were functionalized with PCL nanofibers (Figure 2A) to create a composite scaffold (Figure 2C). SEM revealed randomly oriented nanofibers and their deposition onto the PP mesh. Stereological analyses divided the nanofibers into two fractions of PCL fibers. The first fraction contained fibers with an average diameter of $1.29 \times 10^{3} \pm 0.33 \times 10^{3} \mathrm{~nm}$, while in the second fraction the average diameter was $469 \pm 171 \mathrm{~nm}$. The average diameter of the PP mesh fibers was about $152 \times 10^{3} \pm 5.8 \times 10^{3} \mathrm{~nm}$. This kind of system was therefore considered suitable for further cell studies.
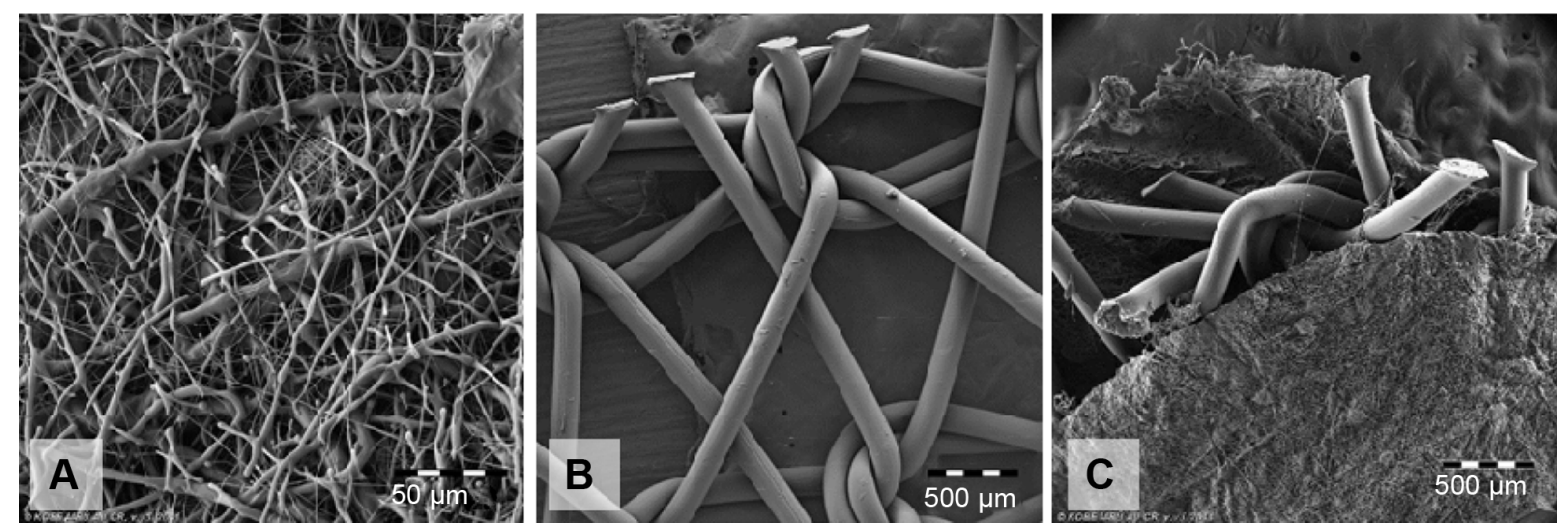

Figure 2 Scanning electron microscopy of the scaffolds. (A) Poly- $\varepsilon$-caprolactone (PCL) nanofibers, (B) polypropylene (PP) mesh, and (C) PP mesh functionalized with PCL nanofibers.

Notes: (A) Magnification $\times 230$, scale bar $50 \mu \mathrm{m}$; (B and C) magnification $\times 18$, scale bar $500 \mu \mathrm{m}$. 


\section{Poly- $\varepsilon$-caprolactone nanofibers} significantly improved cell adhesion and metabolic activity

Functionalization of the PP mesh significantly improved the adhesion and metabolic activity of the $3 \mathrm{~T} 3$ fibroblasts.

First, cell adhesion was evaluated 24 hours after seeding. The $3 \mathrm{~T} 3$ fibroblasts were first stained using DiOC6 and propidium iodide, then visualized using confocal microscopy before areas of the spread cell surface were measured. A significantly larger surface area of the cells was observed on the PP mesh functionalized with PCL nanofibers than on the PP mesh alone (Figure 3 ). In addition, the largest spreading area was for the cells cultivated on the PP mesh functionalized with PCL nanofibers and TRS. Simultaneously, enrichment of the PP mesh with TRS improved cell adhesion, and the cell spreading area of PP + TRS was significantly larger than for PP $(P<0.001)$. Clearly, functionalization of the PP meshes with PCL nanofibers alone, and also treatment of PP meshes with TRS, significantly improved 3T3 adhesion. These two actions seem to be independent, and can therefore result in an additive effect.

Metabolic activity of the 3T3 fibroblasts was determined by MTT assay. The MTT assay revealed significantly higher metabolic activity of the cells after the 10th day of cultivation on all of the composite scaffolds than on the PP mesh alone (Figure 4). Moreover, significantly higher $(P<0.001)$ cell metabolic activity was observed on all of the functionalized composite scaffolds on Day 14.
Significantly higher metabolic activity was also observed on the PP meshes functionalized with PCL nanofibers and the PP meshes functionalized with PCL nanofibers treated with TRS after the 7 th day of cultivation. Additionally, a combination of two improvements to PP mesh - namely, functionalization with PCL nanofibers and treatment with TRS - led to significantly higher $(P<0.001)$ cell metabolic activity on Day 14 than that on all other scaffolds that were used.

\section{Increased metabolic activity is accompanied by a larger number of cells}

The increase in the number of cells, which is the result of good proliferation, was estimated from the DNA values measured using a PicoGreen assay (Figure 5). The results of the PicoGreen assay on Days 1, 3, 7, 10, and 14 clearly indicated substantial cell proliferation on the functionalized scaffolds. A significantly higher number of cells was observed on Days 10 and 14 on each composite scaffold than on the PP mesh alone. Additionally, the PicoGreen assay confirmed a significantly larger number of cells $(P<0.001)$ on the scaffold with the most advanced improvement (ie, the PP mesh functionalized with PCL nanofibers and treated with TRS) on Days 10 and 14 than on all other scaffolds that were investigated. These results are consistent with the results for metabolic activity evaluated by the MTT assay.

However, the concentration of cells does not reflect the ratio of live and dead cells, so a live/dead cell-staining assay

\section{Cell adhesion evaluated on the 1st day after seeding}

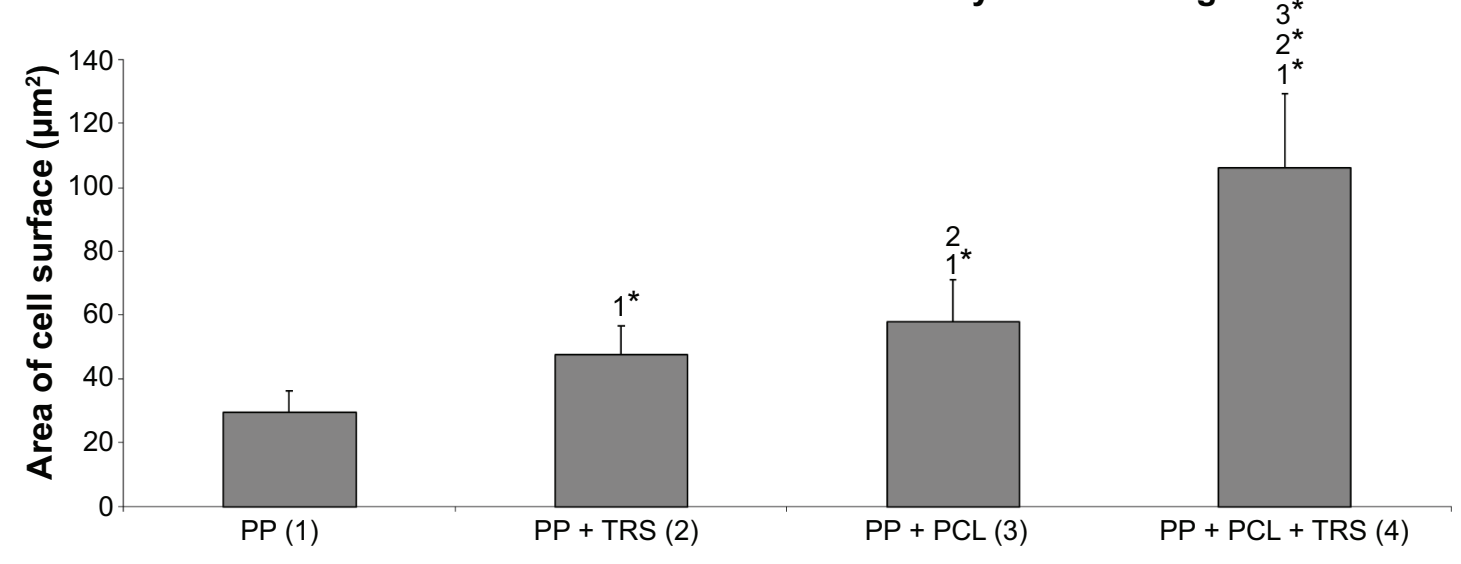

Figure 3 Cell adhesion evaluated on the Ist day after seeding. (I) Average surface area of spread 3 T3 fibroblasts cultivated on the surface of a polypropylene (PP) mesh, (2) a PP mesh treated with thrombocyte-rich solution (TRS), (3) a PP mesh functionalized with poly- $\varepsilon$-caprolactone (PCL) nanofibers, and (4) a PP mesh functionalized with PCL nanofibers treated with TRS. Cell adhesion assay revealed a significantly larger surface area of spread 3T3 fibroblasts on scaffolds functionalized with PCL nanofibers (PP + $P C L$ and PP + PCL + TRS) than on scaffolds without functionalization (PP and PP + TRS). Moreover, the average surface area of 3 T3 fibroblasts was significantly higher (level of significance at a value of $P<0.00 \mathrm{I}$ ) on the PP mesh functionalized with PCL nanofibers treated with TRS than on all other scaffolds.

Notes: The level of statistical significance for the assays is designated above the mean values $(P<0.05$ indicated by number; $P<0.00 \mathrm{I}$ indicated by number and $*)$. Day $\mathrm{I}$ : $2>I^{*} ; 3>I^{*}, 2 ; 4>I^{*}, 2^{*}, 3^{*}$. 


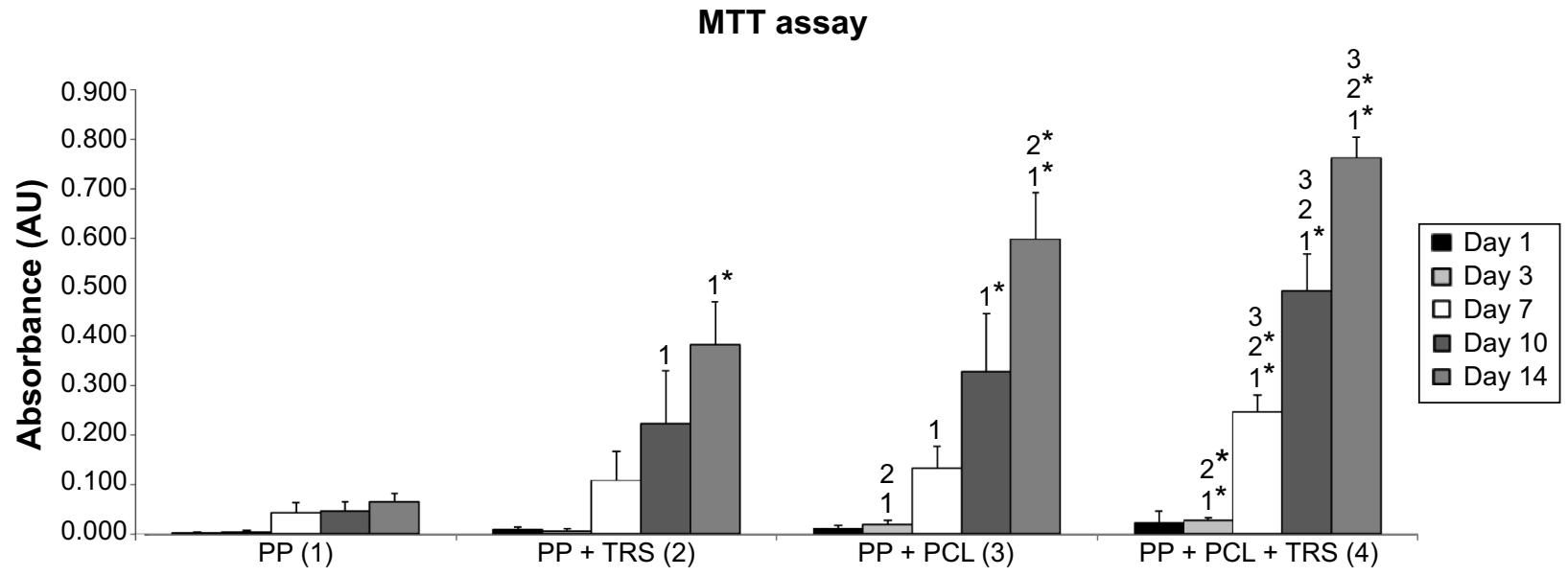

Figure 4 Metabolic activity of 3T3 fibroblasts cultivated on the surface of (I) polypropylene (PP) mesh, (2) PP mesh enriched with adhered thrombocytes, (3) PP mesh functionalized with poly- $\varepsilon$-caprolactone (PCL) nanofibers, and (4) PP mesh functionalized with PCL nanofibers enriched with adhered thrombocytes. 3-[4,5-dimethylthiazol2-yl]-2,5-diphenyltetrazolium bromide (MTT) assay revealed significantly higher metabolic activity of $3 \mathrm{~T} 3$ fibroblasts on scaffolds functionalized with PCL nanofibers (PP + PCL and PP + PCL + thrombocyte-rich solution [TRS]) on Day I4 than on scaffolds without functionalization (PP). Moreover, the metabolic activity of $3 T 3$ fibroblasts on Days 7 , 10 , and 14 was significantly higher on the PP mesh functionalized with PCL nanofibers treated with TRS than on all other scaffolds.

Notes: The level of statistical significance for the assays is designated above the mean values $(P<0.05$ indicated by a number; $P<0.00 \mathrm{I}$ indicated by a number and $*)$. Day I: without significance. Day 3: 3>I, 2; $4>I^{*}, 2^{*}$. Day $7: 3>I ; 4>I^{*}, 2^{*}, 3$. Day $10: 2>I ; 3>I^{*} ; 4>I^{*}, 2,3$. Day $\mid 4: 2>I^{*} ; 3>I^{*}, 2^{*} ; 4>I^{*}, 2^{*}, 3$.

was performed. The viability of the $3 \mathrm{~T} 3$ fibroblasts was evaluated on Days 1, 3, 7, 10, and 14 after seeding. For simplicity, only data obtained on Day 14 are presented (Figure 6). Live cells were stained by BCECF-AM (green color) and by propidium iodide (red). Viability was calculated as the percentage of live cells from the total cell count per unit area. Live/dead cell staining revealed a higher percentage of viable cells on all scaffolds either functionalized with PCL nanofibers or treated with TRS than on a PP mesh alone. In particular, the percentage of viable cells was $85.4 \%$ on the PP mesh treated only with TRS, $88.3 \%$ on the PP mesh functionalized with PCL nanofibers, and $90.1 \%$ on the PP mesh functionalized with PCL nanofibers and treated with TRS. In the two control groups, the percentage of viable cells was $90.3 \%$ for the cells seeded on PCL nanofibers alone, and $94.7 \%$ for the cells seeded on PCL nanofibers treated with TRS. Previous results in this study confirmed the conclusion that a PP mesh enriched by either PCL nanofiber functionalization or TRS treatment improves $3 \mathrm{~T} 3$ fibroblast viability.

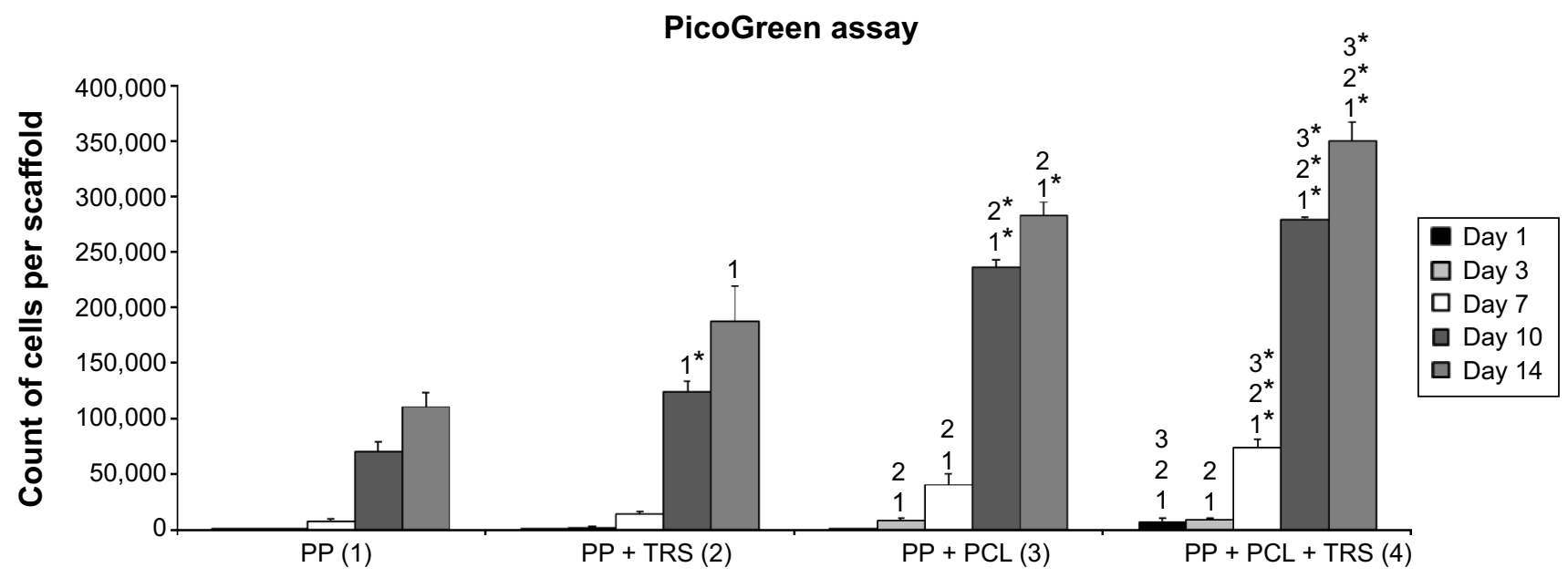

Figure 5 Proliferative activity of 3T3 fibroblasts cultivated on the surface of (I) polypropylene (PP) mesh, (2) PP mesh enriched with adhered thrombocytes, (3) PP mesh functionalized with poly- $\varepsilon$-caprolactone (PCL) nanofibers, and (4) PP mesh functionalized with PCL nanofibers treated with thrombocyte-rich solution (TRS). The PicoGreen ${ }^{\circledR}$ assay revealed significantly higher proliferation of 3 T3 fibroblasts on scaffolds functionalized with PCL nanofibers (PP + PCL and PP + PCL + TRS) on Days 7 , I0, and I4 than on scaffolds without functionalization (PP and PP + TRS). In addition, the proliferation of 3T3 fibroblasts on Days 7, 10, and I4 was significantly higher (level of significance at value of $P<0.00 \mathrm{I})$ on the PP mesh functionalized with $\mathrm{PCL}$ nanofibers enriched with adhered thrombocytes than on all other scaffolds.

Notes: The level of statistical significance for the assays is designated above the mean values $(P<0.05$ indicated by a number; $P<0.00 \mathrm{I}$ indicated by a number and $*)$. Day I: 4>I, 2, 3. Day 3: 3>I, 2; 4>I, 2. Day 7: 3>I, 2; 4>I*, 2*, 3*. Day I0: $2>I^{*} ; 3>I^{*}, 2^{*} ; 4>I^{*}, 2^{*}, 3^{*}$. Day I4: $2>1 ; 3>I^{*}, 2 ; 4>I^{*}, 2^{*}, 3^{*}$. 


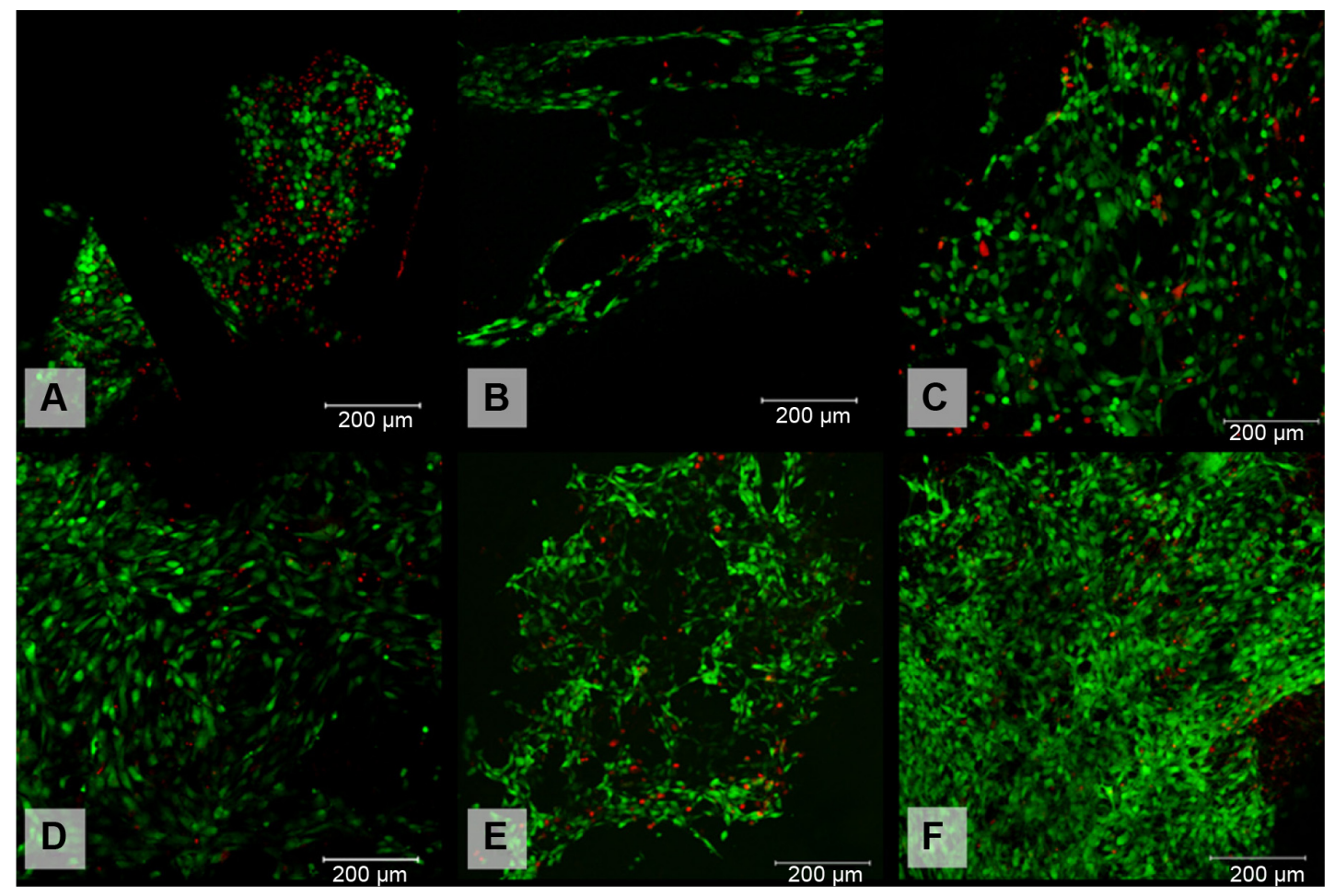

Figure 6 Viability of 3T3 fibroblasts cultivated on the surface of (A) polypropylene (PP) mesh, (B) PP mesh treated with thrombocyte-rich solution (TRS), (C) PP mesh functionalized with poly- $\varepsilon$-caprolactone (PCL) nanofibers, (D) PP mesh functionalized with PCL nanofibers treated with TRS, (E) PCL nanofibers, and (F) PCL nanofibers treated with TRS on Day 14 after seeding. Live/dead cell staining revealed a higher percentage of viable cells on all scaffolds functionalized either with PCL nanofibers or with TRS than on the scaffold without any functionalization or treatment (PP). The percentages of viable cells cultivated on the various surfaces of the scaffolds were: (A) $59.5 \%$, (B) $85.4 \%$, (C) $88.3 \%$, (D) $90.1 \%$, (E) $90.3 \%$, and (F) $94.7 \%$.

Notes: The viability of 3 T3 fibroblasts was evaluated on Days I, 3, 7, 10, and I4 after seeding. For simplification, only data obtained on Day I4 are presented. Viability was calculated as the percentage of live cells from the total cell count per unit area. Live cells are stained green. Dead cells are stained red. Scale bar $200 \mu \mathrm{m}$.

\section{Functionalization of the polypropylene mesh also improved cell proliferation significantly}

Cell metabolic activity can result not only in a larger number of cells, as was confirmed by our study of DNA content, but also an increase in cell proliferation. Cell proliferation was therefore evaluated using a BrdU colorimetric immunoassay. This assay is based on incorporating bromodeoxyuridine only in the active process of DNA synthesis in healthy cells. BrdU colorimetric immunoassay was performed on Days 1, 3, 7, 10, and 14 (Figure 7). The BrdU assay revealed significantly higher proliferation of 3T3 fibroblasts on all evaluation days on scaffolds functionalized with PCL nanofibers and on scaffolds with PCL nanofibers treated with TRS than on scaffolds without functionalization - namely, the PP mesh alone and the PP mesh treated with TRS. Moreover, the proliferation of 3T3 fibroblasts on Day 14 was significantly higher $(P<0.001)$ on the PP mesh functionalized with PCL nanofibers and treated with TRS than on all other scaffolds. Thus, PP mesh functionalization also significantly improved 3 T3 fibroblast proliferation.

\section{Discussion}

Incisional hernia is the most common postoperative complication following abdominal surgery, affecting up to $20 \%$ of patients after midline incision. ${ }^{1,2}$ Insertion of a synthetic material has become the standard of care in ventral hernia repair. However, it has been found that the implementation of a mesh does not reduce the risk of recurrence and only delays it by $2-3$ years. ${ }^{4}$ These observations have emphasized the need to find a new material that is more effective in preventing the formation of an incisional hernia. More than 100 surgical meshes have now been developed, though no ideal mesh exists yet. ${ }^{11}$ The aim of the study reported here was to develop a functionalized scaffold for preventing or repairing incisional hernias.

$\mathrm{PP}$ is the most widely used prosthetic material for repairing ventral hernias. ${ }^{42} \mathrm{~A}$ combination of PCL nanofibers with a PP surgical mesh (Prolene) was chosen as a suitable material for our study. Nowadays, PCL seems to be used more often as a biocompatible soft and hard tissue material, and it also includes a resorbable suture, a drug-delivery system, and bone-graft substitutes, but, as far as we are aware, only 


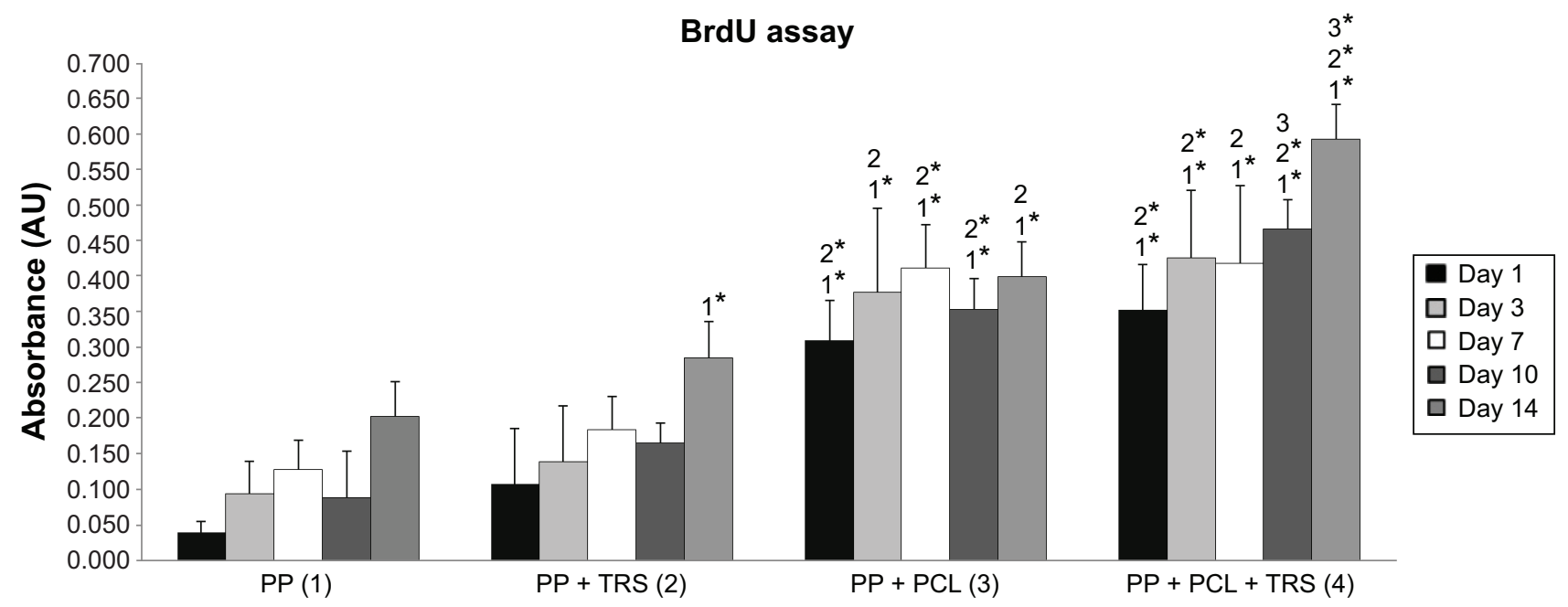

Figure 7 Proliferation of 3 T3 fibroblasts cultivated on the surface of (I) polypropylene (PP) mesh, (2) PP mesh treated with thrombocyte-rich solution (TRS), (3) PP mesh functionalized with poly- $\varepsilon$-caprolactone $(\mathrm{PCL})$ nanofibers, and (4) PP mesh functionalized with PCL nanofibers treated with TRS. A 5-bromo-2'-deoxyuridine (BrdU) colorimetric immunoassay revealed significantly greater proliferation of $3 T 3$ fibroblasts on scaffolds functionalized with $P C L$ nanofibers $(P P+P C L$ and $P P+P C L+T R S)$ on all days of evaluation than on scaffolds without functionalization (PP and PP + TRS). In addition, the proliferation of 3 T3 fibroblasts on Day I4 was significantly higher ( $<0.00 \mathrm{I})$ on the PP mesh functionalized with PCL nanofibers treated with TRS than on all other scaffolds.

Notes: The level of statistical significance for the assays is designated above the mean values $(P<0.05$ indicated by a number, $P<0.00 \mathrm{I}$ indicated by a number and $*)$. Day I: 3>I*, $2^{*} ; 4>I^{*}, 2^{*}$. Day 3: 3>I*, 2; $4>I^{*}, 2^{*}$. Day $7: 3>I^{*}, 2^{*} ; 4>I^{*}, 2$. Day $10: 3>I^{*}, 2^{*} ; 4>I^{*}, 2^{*}, 3$. Day $14: 2>I^{*} ; 3>I^{*}, 2 ; 4>I^{*}, 2^{*}, 3^{*}$.

Abbreviation: $\mathrm{AU}$, absorbance units.

a few studies have investigated PCL for ventral hernia repairs. ${ }^{25,43}$ Guillaume et al used PCL as a coating agent and a drug reservoir for anti-infective drugs by heating deposition onto PP prostheses. ${ }^{44}$ Another study using PCL in the form of nanofibers as a carrier for antibiotics was evaluated by Bölgen et al ${ }^{45}$ The antibiotic-embedded PCL membranes eliminated postsurgery adhesions and improved healing in rats. In the most recent study, Zhao et al used an electrospun PCL/collagen hybrid scaffold for congenital diaphragmatic hernia reconstruction in rats. The aligned scaffolds allowed muscle cell migration and tissue formation. ${ }^{46}$

In general, nanofibers have recently been used in various tissue-engineering applications. ${ }^{47}$ The advantages of using nanofibers for hernia regeneration lie in the simplicity of the process for preparing submicron-scale fibers and their low cost. In addition, manufactured nanofibers can easily be modified and functionalized to refine their biological and biomechanical properties. Various studies have proved that nanofibers - namely, PCL nanofibers - support the adhesion, growth, and proliferation of fibroblasts, chondrocytes, and MSCs. ${ }^{23,28,31}$ Moreover, PCL nanofibers possess suture retention and tensile strength appropriate for most hernia repairs. The studies of Ebersole et $\mathrm{al}^{12}$ and Deeken et $\mathrm{al}^{48}$ showed that electrospun scaffolds having mechanical properties within the predefined range may be suitable for further evaluation in preclinical trials. Our previous study proved the suitability of a composite scaffold based on PCL nanofibers deposited on a PP mesh for hernia regeneration in rabbits. ${ }^{29}$ A histological and biomechanical evaluation revealed PCL nanofibers had better healing capacity than conventional PP mesh for preventing hernia formation. ${ }^{29}$

In the present study, we tested a new composite scaffold based on a PP surgical mesh, PCL nanofibers, and adhered thrombocytes. We evaluated the effect of released GFs from thrombocytes and the effect of PCL nanofiber functionalization on 3T3 fibroblast proliferation in vitro. For this study, 3T3 fibroblasts were chosen as a biocompatibility model system. In extensive in vitro tests, we have proven that PCL nanofibers have excellent biocompatible properties. We have confirmed that PCL nanofibers deposited on a PP mesh promote the better adhesion, growth, metabolic activity, proliferation, and viability of 3T3 fibroblasts in all tests than a PP mesh alone.

The advanced improvement of the composite scaffolds tested in vitro seems to be promising for regenerating ventral or incisional hernias. We used a simple drug-delivery system, developed by our group, based on thrombocytes adhered onto PCL nanofibers. ${ }^{23}$ The approaches that have been used so far, in which simple GFs or thrombocytes are administered, may tend to diffuse active substances away from the site of the injury. ${ }^{49}$ The problem with diffusing of active substances away can be solved by using suitable biocompatible scaffolds to which the desired drugs could be attached. One of the 
first attempts to set up a dual drug-delivery system based on polymeric scaffolds was reported by Richardson et al. ${ }^{50}$ Better control of either drug or GF pharmacokinetics could be achieved by using a combination of active substances with natural or synthetic biomaterials, such as collagen or PCL composite scaffolds. ${ }^{51}$ Previous studies have mentioned various drug-delivery systems based on a PCL composite scaffold successfully used for hernia regeneration. ${ }^{29,44,45}$

PCL nanofibers were used in our study as a carrier of GFs gradually released from platelets adhered to the nanofiber surface, with the aim of using the material in hernia regeneration. The use of this system enabled us to avoid the complications caused by a burst release of high doses of GFs. In addition, the GFs were prevented from proteolysis and loss of bioactivity both by the scaffold and the platelets themselves. ${ }^{52}$ This enables a prolonged therapeutic effect of GFs. GFs naturally occurring in platelets have been shown to be effective in promoting wound healing and regeneration. ${ }^{38}$ Platelets in the form of TRS or PRP have been playing a rapidly growing role in tissue engineering. ${ }^{53}$ For an evaluation of the possible positive effect of PCL nanofibers and GFs in the form of adhered platelets for ventral hernia repair, we used $3 \mathrm{~T} 3$ fibroblasts in extensive in vitro tests. The positive effect of PRP on fibroblast proliferation has already been described by Anitua et al, ${ }^{54}$ Creeper and Ivanovski, ${ }^{55}$ and Passaretti et al. ${ }^{56}$ Unfortunately these teams did not use a polymeric scaffold, so it was not possible to observe the benefits from the multiplication of the positive effect on the fibroblasts from both the polymer scaffold and the PRP. Platelets were shown to release numerous GFs with mitogenic and anabolic function (eg, platelet-derived growth factor, epidermal growth factor, and transforming growth factor-beta). ${ }^{36,37}$ Our observation of increased proliferation, metabolic activity, and DNA synthesis of the 3T3 fibroblasts in TRS-containing sample groups in the present study is explained by stimulatory effect of such active molecules. In addition, upon activation, platelets form a protein layer consisting of fibrinogen, von Willebrand factor, and other proteins, ${ }^{57}$ which, together with the PCL nanofibers mimicking ECM, has a possible stimulatory effect on cell adhesion. Moreover, we used in our study TRS, which does not include leukocytes and erythrocytes in comparison to PRP obtained from whole blood. Prosecká et al showed that even human TRS promotes tissue regeneration in a rabbit model with a minimal immunological negative response. ${ }^{58}$ This kind of result favors TRS for further preclinical and clinical studies.

\section{Conclusion}

Our experiments prove a positive effect of PCL nanofibers deposited on a PP mesh on the adhesion, growth, metabolic activity, proliferation, and viability of 3T3 fibroblasts. The positive effect is multiplied by using a simple drug-delivery system in the form of adhered thrombocytes. The results presented in our study show the potential of this system for ventral or incisional hernia regeneration.

\section{Acknowledgments}

This work was supported by the Academy of Sciences of the Czech Republic (the Institutional Research Concept RVO 68378041), the Ministry of Education Youth and Sports of the Czech Republic (project IPv6), the Grant Agency of the Charles University (grant numbers 545313, 384311, 270513, 424213, and 648112), the Internal Grant Agency of the Ministry of Health of the Czech Republic (grant number NT12156), and University Hospital Motol (grant number 9775).

The authors would like to acknowledge O Kofroňová and $\mathrm{O}$ Benada from the Laboratory of Molecular Structure Characterization, Institute of Microbiology, Academy of Sciences of the Czech Republic, Prague, Czech Republic, for performing SEM analyses.

\section{Disclosure}

The authors report no conflicts of interest in this work.

\section{References}

1. Sugerman HJ, Kellum JM Jr, Reines HD, DeMaria EJ, Newsome HH, Lowry JW. Greater risk of incisional hernia with morbidly obese than steroid-dependent patients and low recurrence with prefascial polypropylene mesh. Am J Surg. 1996;171(1):80-84.

2. Höer J, Lawong G, Klinge U, Schumpelick V. [Factors influencing the development of incisional hernia. A retrospective study of 2,983 laparotomy patients over a period of 10 years.] Chirurg. 2002;73(5):474-480. German.

3. Burger JW, Luijendijk RW, Hop WC, Halm JA, Verdaasdonk EG, Jeekel J. Long-term follow-up of a randomized controlled trial of suture versus mesh repair of incisional hernia. Ann Surg. 2004;240(4):578-583; discussion 583-575.

4. Flum DR, Horvath K, Koepsell T. Have outcomes of incisional hernia repair improved with time? A population-based analysis. Ann Surg. 2003; 237(1):129-135.

5. Vrijland WW, Bonthuis F, Steyerberg EW, Marquet RL, Jeekel J, Bonjer HJ. Peritoneal adhesions to prosthetic materials: choice of mesh for incisional hernia repair. Surg Endosc. 2000;14(10): 960-963.

6. Jacob BP, Hogle NJ, Durak E, Kim T, Fowler DL. Tissue ingrowth and bowel adhesion formation in an animal comparative study: polypropylene versus Proceed versus Parietex Composite. Surg Endosc. 2007;21(4):629-633.

7. Losanoff JE, Richman BW, Jones JW. Entero-colocutaneous fistula: a late consequence of polypropylene mesh abdominal wall repair: case report and review of the literature. Hernia. 2002;6(3):144-147. 
8. Jezupovs A, Mihelsons M. The analysis of infection after polypropylene mesh repair of abdominal wall hernia. World J Surg. 2006;30(12): 2270-2278; discussion 2279-2280.

9. Usher FC, Ochsner J, Tuttle LL Jr. Use of marlex mesh in the repair of incisional hernias. Am Surg. 1958;24(12):969-974.

10. Luijendijk RW, Hop WC, van den Tol MP, et al. A comparison of suture repair with mesh repair for incisional hernia. $N$ Engl J Med. 2000;343(6):392-398.

11. Shankaran V, Weber DJ, Reed RL 2nd, Luchette FA. A review of available prosthetics for ventral hernia repair. Ann Surg. 2011;253(1): 16-26.

12. Ebersole GC, Buettmann EG, MacEwan MR, et al. Development of novel electrospun absorbable polycaprolactone (PCL) scaffolds for hernia repair applications. Surg Endosc. 2012;26(10):2717-2728.

13. Klinge U, Klosterhalfen B. Modified classification of surgical meshes for hernia repair based on the analyses of 1,000 explanted meshes. Hernia. 2012;16(3):251-258.

14. Coda A, Lamberti R, Martorana S. Classification of prosthetics used in hernia repair based on weight and biomaterial. Hernia. 2012; 16(1):9-20.

15. Burger JW, Halm JA, Wijsmuller AR, ten Raa S, Jeekel J. Evaluation of new prosthetic meshes for ventral hernia repair. Surg Endosc. 2006; 20(8):1320-1325.

16. Catena F, Ansaloni L, Gazzotti F, et al. Use of porcine dermal collagen graft (Permacol) for hernia repair in contaminated fields. Hernia. 2007;11(1):57-60.

17. Butler CE, Prieto VG. Reduction of adhesions with composite AlloDerm/polypropylene mesh implants for abdominal wall reconstruction. Plast Reconstr Surg. 2004;114(2):464-473.

18. Sato T, Chen G, Ushida T, et al. Evaluation of PLLA-collagen hybrid sponge as a scaffold for cartilage tissue engineering. Mater Sci Eng C Mater Biol Appl. 2004;24(3):365-372.

19. Khil MS, Bhattarai SR, Kim HY, Kim SZ, Lee KH. Novel fabricated matrix via electrospinning for tissue engineering. J Biomed Mat Res B Appl Biomater. 2005;72(1):117-124.

20. Kim SH, Turnbull J, Guimond S. Extracellular matrix and cell signalling: the dynamic cooperation of integrin, proteoglycan and growth factor receptor. J Endocrinol. 2011;209(2):139-151.

21. Kim TG, Park TG. Surface functionalized electrospun biodegradable nanofibers for immobilization of bioactive molecules. Biotechnol Prog. 2006;22(4):1108-1113.

22. Jayakumar R, Prabaharan M, Nair SV, Tamura H. Novel chitin and chitosan nanofibers in biomedical applications. Biotechnol Adv. 2010;28(1):142-150.

23. Jakubova R, Mickova A, Buzgo M, et al. Immobilization of thrombocytes on PCL nanofibres enhances chondrocyte proliferation in vitro. Cell Prolif. 2011;44(2):183-191.

24. Hutmacher DW, Schantz T, Zein I, Ng KW, Teoh SH, Tan KC. Mechanical properties and cell cultural response of polycaprolactone scaffolds designed and fabricated via fused deposition modeling. J Biomed Mater Res. 2001;55(2):203-216.

25. Kweon H, Yoo MK, Park IK, et al. A novel degradable polycaprolactone networks for tissue engineering. Biomaterials. 2003;24(5): 801-808.

26. Thapa A, Webster TJ, Haberstroh KM. Polymers with nano-dimensional surface features enhance bladder smooth muscle cell adhesion. J Biomed Mater Res A. 2003;67(4):1374-1383.

27. Venugopal J, Ramakrishna S. Biocompatible nanofiber matrices for the engineering of a dermal substitute for skin regeneration. Tissue Eng 2005;11(5-6):847-854.

28. Chen M, Patra PK, Warner SB, Bhowmick S. Role of fiber diameter in adhesion and proliferation of NIH 3T3 fibroblast on electrospun polycaprolactone scaffolds. Tissue Eng. 2007;13(3):579-587.

29. Plencner M, East B, Tonar Z, et al. Abdominal closure reinforcement by using polypropylene mesh functionalized with poly- $\varepsilon$-caprolactone nanofibers and growth factors for prevention of incisional hernia formation. Int J Nanomedicine. 2014;9:3263-3277.
30. Williamson MR, Adams EF, Coombes AG. Gravity spun polycaprolactone fibres for soft tissue engineering: interaction with fibroblasts and myoblasts in cell culture. Biomaterials. 2006;27(7):1019-1026.

31. Rampichová M, Chvojka J, Buzgo M, et al. Elastic three-dimensional poly ( $\varepsilon$-caprolactone) nanofibre scaffold enhances migration, proliferation and osteogenic differentiation of mesenchymal stem cells. Cell Prolif. 2013;46(1):23-37.

32. Venugopal JR, Zhang Y, Ramakrishna S. In vitro culture of human dermal fibroblasts on electrospun polycaprolactone collagen nanofibrous membrane. Artif Organs. 2006;30(6):440-446.

33. Hromadka M, Collins JB, Reed C, et al. Nanofiber applications for burn care. J Burn Care Res. 2008;29(5):695-703.

34. Buzgo M, Jakubova R, Mickova A, et al. Time-regulated drug delivery system based on coaxially incorporated platelet $\alpha$-granules for biomedical use. Nanomedicine (Lond). 2013;8(7):1137-1154.

35. Drengk A, Zapf A, Stürmer EK, Stürmer KM, Frosch KH. Influence of platelet-rich plasma on chondrogenic differentiation and proliferation of chondrocytes and mesenchymal stem cells. Cells, tissues, organs. 2009;189(5):317-326

36. Nikolidakis D, Jansen JA. The biology of platelet-rich plasma and its application in oral surgery: literature review. Tissue Eng Part B Rev 2008;14(3):249-258.

37. Plachokova AS, Nikolidakis D, Mulder J, Jansen JA, Creugers NH. Effect of platelet-rich plasma on bone regeneration in dentistry: a systematic review. Clin Oral Implants Res. 2008;19(6):539-545.

38. Nauta A, Gurtner GC, Longaker MT. Wound healing and regenerative strategies. Oral Dis. 2011;17(6):541-549.

39. Lukáš D, Sarkar A, Martinová L, et al. Textiles Progress. Vol 41(2), Physical principles of electrospinning (electrospinning as a nano-scale technology of the twenty-first century). Abingdon: Taylor and Francis; 2009.

40. Baenziger NL, Brodie GN, Majerus PW. A thrombin-sensitive protein of human platelet membranes. Proc Natl Acad Sci US A. 1971;68(1): 240-243.

41. Mickova A, Buzgo M, Benada O, et al. Core/Shell nanofibers with embedded liposomes as a drug delivery system. Biomacromolecules. 2012;13(4):952-962.

42. Cobb WS, Peindl RM, Zerey M, Carbonell AM, Heniford BT. Mesh terminology 101. Hernia. 2009;13(1):1-6.

43. Williams JM, Adewunmi A, Schek RM, et al. Bone tissue engineering using polycaprolactone scaffolds fabricated via selective laser sintering. Biomaterials. 2005;26(23):4817-4827.

44. Guillaume O, Lavigne JP, Lefranc O, Nottelet B, Coudane J, Garric X. New antibiotic-eluting mesh used for soft tissue reinforcement. Acta Biomater. 2011;7(9):3390-3397.

45. Bölgen N, Vargel I, Korkusuz P, Menceloğlu YZ, Pişkin E. In vivo performance of antibiotic embedded electrospun PCL membranes for prevention of abdominal adhesions. J Biomed Mater Res B Appl Biomater. 2007;81(2):530-543.

46. Zhao W, Ju YM, Christ G, Atala A, Yoo JJ, Lee SJ. Diaphragmatic muscle reconstruction with an aligned electrospun poly( $\varepsilon$-caprolactone)/ collagen hybrid scaffold. Biomaterials. 2013;34(33):8235-8240.

47. Ma Z, Kotaki M, Inai R, Ramakrishna S. Potential of nanofiber matrix as tissue-engineering scaffolds. Tissue Eng. 2005;11(1-2):101-109.

48. Deeken CR, Abdo MS, Frisella MM, Matthews BD. Physicomechanical evaluation of absorbable and nonabsorbable barrier composite meshes for laparoscopic ventral hernia repair. Surg Endosc. 2011;25(5):1541-1552.

49. Tabata II. The importance of drug delivery systems in tissue engineering. Pharm Sci Technolo Today. 2000;3(3):80-89.

50. Richardson TP, Peters MC, Ennett AB, Mooney DJ. Polymeric system for dual growth factor delivery. Nat Biotechnol. 2001;19(11):1029-1034.

51. Rai B, Teoh SH, Ho KH. An in vitro evaluation of PCL-TCP composites as delivery systems for platelet-rich plasma. J Control Release. 2005;107(2):330-342.

52. Babensee JE, McIntire LV, Mikos AG. Growth factor delivery for tissue engineering. Pharm Res. 2000;17(5):497-504. 
53. Tonti GA, Mannello F. From bone marrow to therapeutic applications: different behaviour and genetic/epigenetic stability during mesenchymal stem cell expansion in autologous and foetal bovine sera? Int $J$ Dev Biol. 2008;52(8):1023-1032.

54. Anitua E, Sánchez M, Zalduendo MM, et al. Fibroblastic response to treatment with different preparations rich in growth factors. Cell Prolif. 2009;42(2):162-170.

55. Creeper F, Ivanovski S. Effect of autologous and allogenic plateletrich plasma on human gingival fibroblast function. Oral Dis. 2012; 18(5):494-500.

56. Passaretti F, Tia M, D'Esposito V, et al. Growth-promoting action and growth factor release by different platelet derivatives. Platelets. 2014;25(4):252-256.

57. Blair P, Flaumenhaft R. Platelet alpha-granules: basic biology and clinical correlates. Blood Rev. 2009;23(4):177-189.
58. Prosecká E, Rampichová M, Litvinec A, et al. Collagen/hydroxyapatite scaffold enriched with polycaprolactone nanofibers, thrombocyte-rich solution and mesenchymal stem cells promotes regeneration in large bone defect in vivo. J Biomed Mater Res A. 2014. Epub May 16.

\section{Publish your work in this journal}

The International Journal of Nanomedicine is an international, peerreviewed journal focusing on the application of nanotechnology in diagnostics, therapeutics, and drug delivery systems throughout the biomedical field. This journal is indexed on PubMed Central, MedLine, CAS, SciSearch ${ }^{\circledR}$, Current Contents ${ }^{\circledR} /$ Clinical Medicine,
Journal Citation Reports/Science Edition, EMBase, Scopus and the Elsevier Bibliographic databases. The manuscript management system is completely online and includes a very quick and fair peer-review system, which is all easy to use. Visit http://www.dovepress.com/ testimonials.php to read real quotes from published authors. 